\title{
A Local Superlens
}

Susanne C. Kehr ${ }^{\star}{ }^{1}$, Raymond G. P. McQuaid ${ }^{2}$, Lisa Ortmann ${ }^{1}$, Thomas Kämpfe ${ }^{1}$, Frederik Kuschewski ${ }^{1}$, Denny Lang ${ }^{1,3}$, Jonathan Döring ${ }^{1}$, J. Marty Gregg ${ }^{2}$, Lukas M. Eng ${ }^{1}$

${ }^{1}$ Institute of Applied Physics/Photophysics, Technische Universität Dresden, 01062 Dresden, Germany

${ }^{2}$ Centre for Nanostructured Media, School of Maths and Physics, Queen's University Belfast, Belfast BT71NN, United Kingdom

${ }^{3}$ Helmholtz-Zentrum Dresden-Rossendorf, Institute of Ion Beam Physics and Materials Research, Bautzner Landstraße 400, 01328 Dresden, Germany

\section{SUPPORTING INFORMATION}

This supporting information includes additional theoretical considerations (Figure S1) as well as complementary data to Figure 4 of the main manuscript (Figures S2-S4). 
(a)

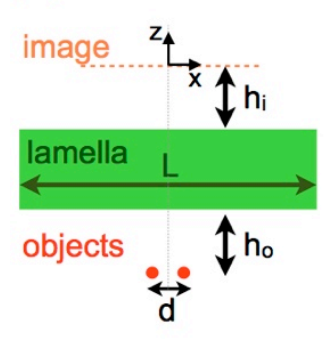

(b)

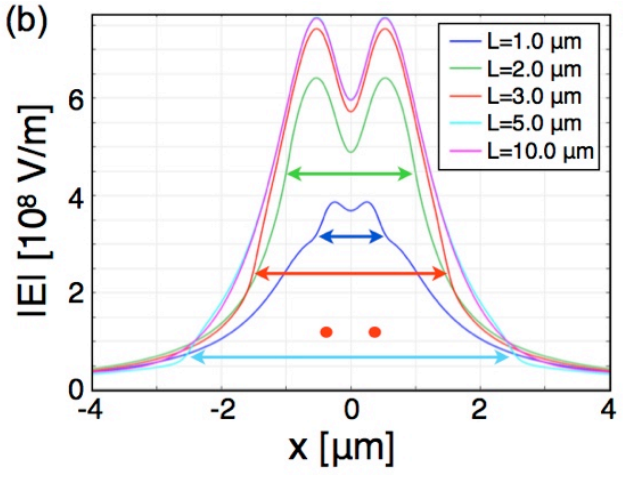

(c)

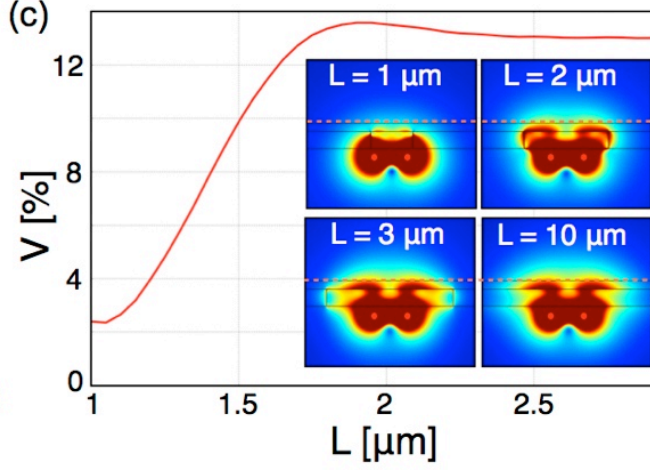

Figure S1 I Role of lateral superlens' dimension. (a) Sample sketch with definition of parameters including lateral size of the superlens lamella $L$ (thickness $400 \mathrm{~nm}$ ), position $h_{0}=200 \mathrm{~nm}$ and distance $d=0.75 \mu \mathrm{m}$ of the two point-dipole objects, as well as position of the image line at $h_{i}=200 \mathrm{~nm}$ at which the electric fields in (b) are recorded. (b) Electric fields along the image line defined in (a) for different lamella sizes $L$ (indicated by arrows). For $L<3 \mu \mathrm{m}$, the signal is reduced and the image is distorted. (c) Contrast $V$ between field maximum and center minimum as a function of $L$ together with field plots (insets). For superlenses smaller than $L=3.3 \mathrm{~d}$ (corresponding to $\lambda / 6.6$ ), the contrast is altered. For $\mathrm{L}<2 \mu \mathrm{m}$ the contrast is strongly reduced and the corresponding field plots illustrate that the lens does not reconstruct the object information any more.

In Figure S1 we discuss the influence of the superlens' lateral size by numerical simulations using the commercial finite-element solver COMSOL 5.0. Here, we assume a system consisting of a BTO-lamella superlens (thickness of $400 \mathrm{~nm}$, lateral dimension $L$ ) and two point sources separated by $d=0.75 \mu \mathrm{m}$ and located at a distance $h_{0}=200 \mathrm{~nm}$ to the lamella surface (see Fig. S1a). The electric field distribution along the image line at a distance $h_{i}=200 \mathrm{~nm}$ to the slab is displayed in Fig. S1b and depends on the lateral dimension $L$ of the lamella. For an extended slab, one can clearly separate the two line sources in the image plane, which is maintained down to a finite sizes of $L=3 \mu \mathrm{m}$. For $L<3 \mu \mathrm{m}$ the image is altered and the overall signal intensity is reduced. Here, we define the contrast $V=\left(E_{\max }-\right.$ $\left.E_{\min }\right) /\left(E_{\max }+E_{\min }\right)$ with $E_{\max }$ and $E_{\min }$ being the field's maximum and center minimum along the image line, respectively. In Figure S1c, we show the contrast $V$ as a function of $L$ as well as selected field plots. Here we observe a constant contrast of $V_{\infty}=13 \%$ for $L=2.5 \mu \mathrm{m}$ to infinity. For $2 \mu \mathrm{m}<L<2.5 \mu \mathrm{m}$, the contrast is slightly enhanced while for $L<2 \mu \mathrm{m}$ it rapidly drops down to $V=2 \%$ for $L=1 \mu \mathrm{m}$. This behavior can be explained by looking at the electric field in the inserted field plots: For $L>2.5 \mu \mathrm{m}$, the superlens creates an undistorted image of the objects' field with a reduced field strength due to absorption of the material. For $L<2.5 \mu \mathrm{m}$, the image is still maintained, however, the edges of the superlens scatter additional fields into the image that enhance the overall signal at the sides resulting in an enhanced contrast. For $L<2 \mu \mathrm{m}$, the electric field from the objects is not only exciting the lower interface of the lamella, but also its sides. Hence, additional fields at the superlens surface reduce the image contrast and cause a blurring and distortion of the image. Moreover scattering at the edges causes a sub-structure that is rather attributed to the shape of the superlens than to the object distribution. In conclusion, these theoretical results show a non-distorted superlens image for subwavelength-sized superlenses down to $L=2.5 \mu \mathrm{m}$ corresponding to $L=\lambda / 6.6$. However, in order to create a non-distorted image, the lamella should be significantly larger than the object distribution $d$ which is in our example $L=2.5 \mu \mathrm{m}=3.3 \mathrm{~d}$. 
(a)
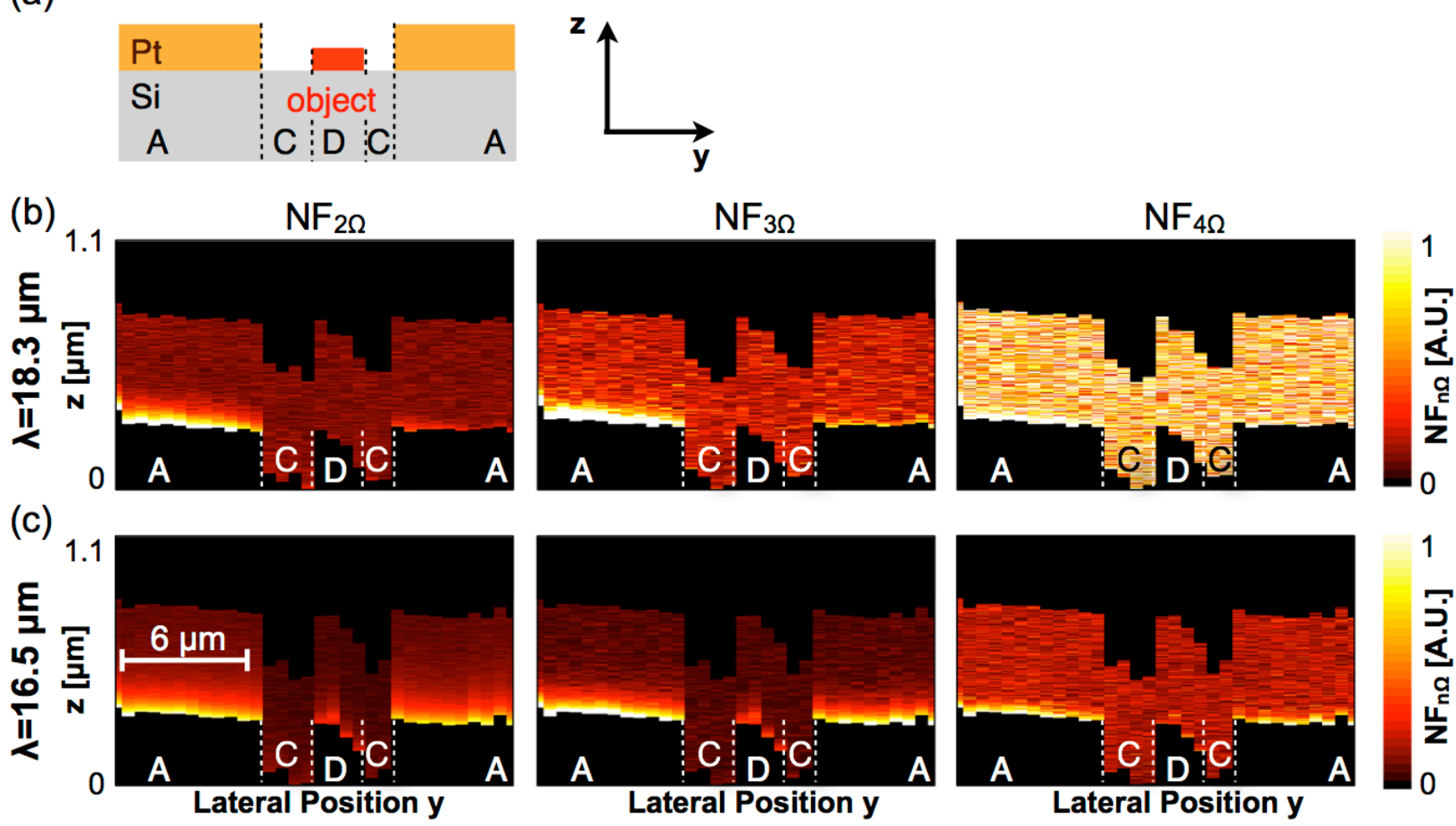

Figure S2 I Near-field cross sections. (a) Sample sketch with areas on Pt film (A), Si substrate (C), and $\mathrm{Pt}$ objects within the trench (D). (b,c) All images show near-field data (normalized to the laser power) displayed in color-coding as a function of distance $z$ and the lateral position $x$ on the sample. Here, the reference structure of the sample is scanned along a line crossing the square-shaped dot within the gap in the center (area (D) in (a), also see Fig. 1d). From left to right, near-field signals are demodulated at increasing higher harmonic frequencies $\mathrm{n} \Omega$ displayed in the same color scales for the same harmonic signals. As none of the structures shows a material resonance, the behavior at both wavelengths of Fig. 4, namely (a) $\lambda=18.3 \mu \mathrm{m}$ and (b) $\lambda=16.5 \mu \mathrm{m}$, reflect a simple, evanescent decay of the signal that is uniform on all Pt areas [areas (A) and (D)] and dark on the silicon substrate (C) within the gap. Compared to the extended Pt film (A) the reference dot (D) appears somewhat darker as it is already subwavelength in size and, hence, shows a less intense near-field response. Please note that for the 4th harmonic signal and $\lambda=18.3 \mu \mathrm{m}$ [far right in (b)], the near-field signal lies within the noise level of the detector.

In Figure S2 we display near-field cross sections on the reference structure at wavelengths corresponding to on- and off-resonant excitations of the BTO superlens (see Fig. 3 of main manuscript). Compared to the superlens, the reference structure consists of platinum (plain film in area (A) and structured dots (D) in the trench) and the silicon substrate (area (C) in the gap) and, hence, shows no material resonances in the wavelength range of examination. Consequently, the behavior at both displayed wavelengths at $\lambda=18.3 \mu \mathrm{m}$ and $\lambda=16.5 \mu \mathrm{m}$, is very similar and shows the typical evanescent decay of near-field signals over a distance of $200 \mathrm{~nm}$ on all regions with platinum $[(A)$ and $(D)$ elevated in topography]. Within the gap on the silicon substrate (C), we basically observe no near-field signal, as silicon is transparent in this wavelength regime. The reference dot (D) appears somewhat darker compared to the Pt film (A) as it is subwavelength-sized and non-resonant, and, hence, its near-field response is reduced. 
Moreover, near-field cross sections on both the lamella superlens (Fig. S3) and the reference dots (Fig. S4) are shown for various wavelengths between $15.7 \mu \mathrm{m}$ and $18.3 \mu \mathrm{m}$ and different harmonic demodulation at $2 \Omega, 3 \Omega$, and $4 \Omega$. This data can be compared directly with Figures 3 and S2 showing the same dimensions and color scales.

The additional data on the lamella superlens (Fig. S3) complement the data displayed in Fig. 4 of the main manuscript. In the wavelength range between $15.7 \mu \mathrm{m}$ and $18.3 \mu \mathrm{m}$ two different modes in the sample are excited (see Figs. 2 and 5), namely top-layer polariton mode and coupling of polariton-modes at the two interfaces of the lamella with the latter leading to the superlensing effect. Superlensing appears most distinct in the 3rd and 4th harmonic near-field signals when far-field effects are sufficiently suppressed. Here, superlensing appears between $15.9 \mu \mathrm{m}$ and $16.9 \mu \mathrm{m}$ [Fig. S3 - Part $2-(\mathrm{g})-(\mathrm{l})$ ] showing the image of the Pt structure as a bright maximum in the center of the lamella (D). For longer wavelengths between $17 \mu \mathrm{m}$ and $18.3 \mu \mathrm{m}$ [Fig. S3 - Part $1-(\mathrm{a})$-(f)] the top-layer polariton mode results in an enhanced near-field signal on the whole lamella showing no clear image of the underlying structure.

In Figure S4 we complement these results with measurements on the reference structure showing a non-resonant response with strong near-field signal on all areas with platinum surface (A) and (D) similar to the behavior discussed in Figure S2 but for a wider wavelength range. 

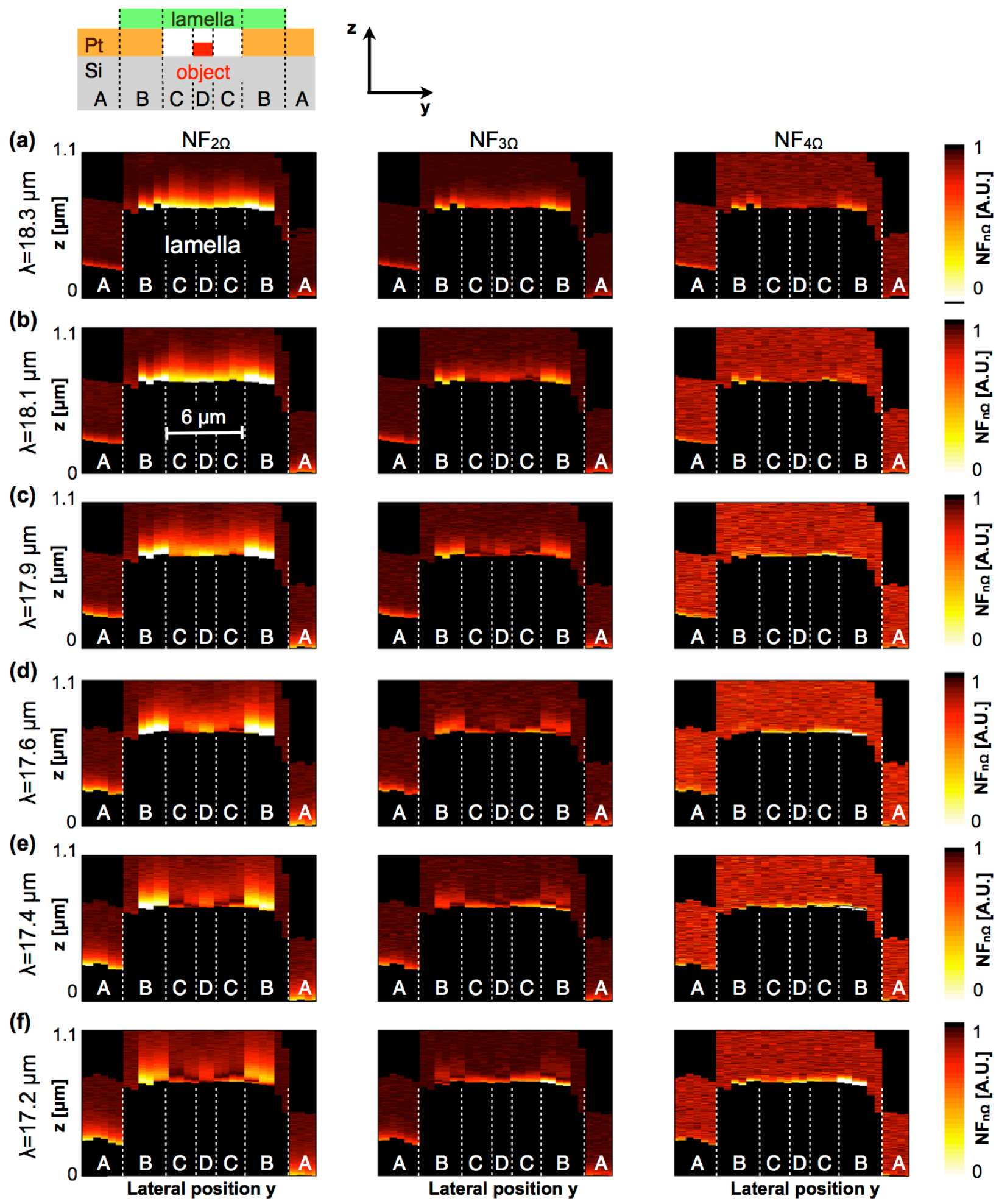

Figure S3 - Part 1 I Near-field cross sections along the lamella superlens (areas (B)-(D), elevated area in the center). For wavelengths between (a) $18.3 \mu \mathrm{m}$ to (f) $17.2 \mu \mathrm{m}$ no distinct superlensing is observed, but the lamella appears bright in areas (B)-(D) due to a polariton-enhanced near-field resonance. All near-field data were normalized to the incident laser power and are displayed in the same color scales for the same harmonics. 

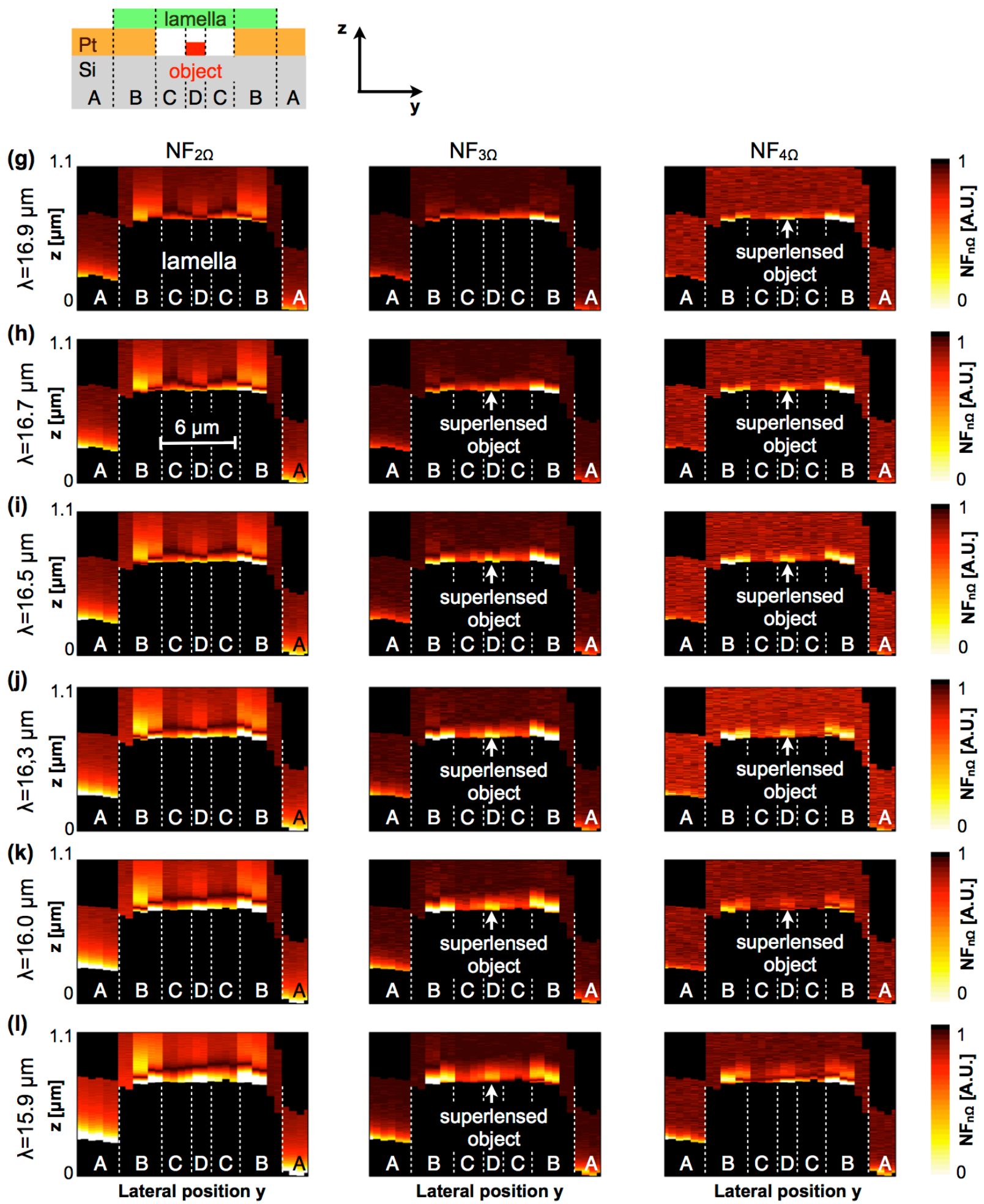

Figure S3 - Part 2 I Near-field cross sections along the lamella superlens (areas (B)-(D), elevated area in the center). For wavelengths between (g) $16.9 \mu \mathrm{m}$ to (I) $15.9 \mu \mathrm{m}$ the objects below the lamella superlens become observable on its image side [area (D)] in particular in the $3^{\text {rd }}$ and $4^{\text {th }}$ harmonic near-field signals. The image becomes most distinct at $\lambda=16.5 \mu \mathrm{m}$ (i). All near-field data were normalized to the incident laser power and are displayed in the same color scales for the same harmonics. 

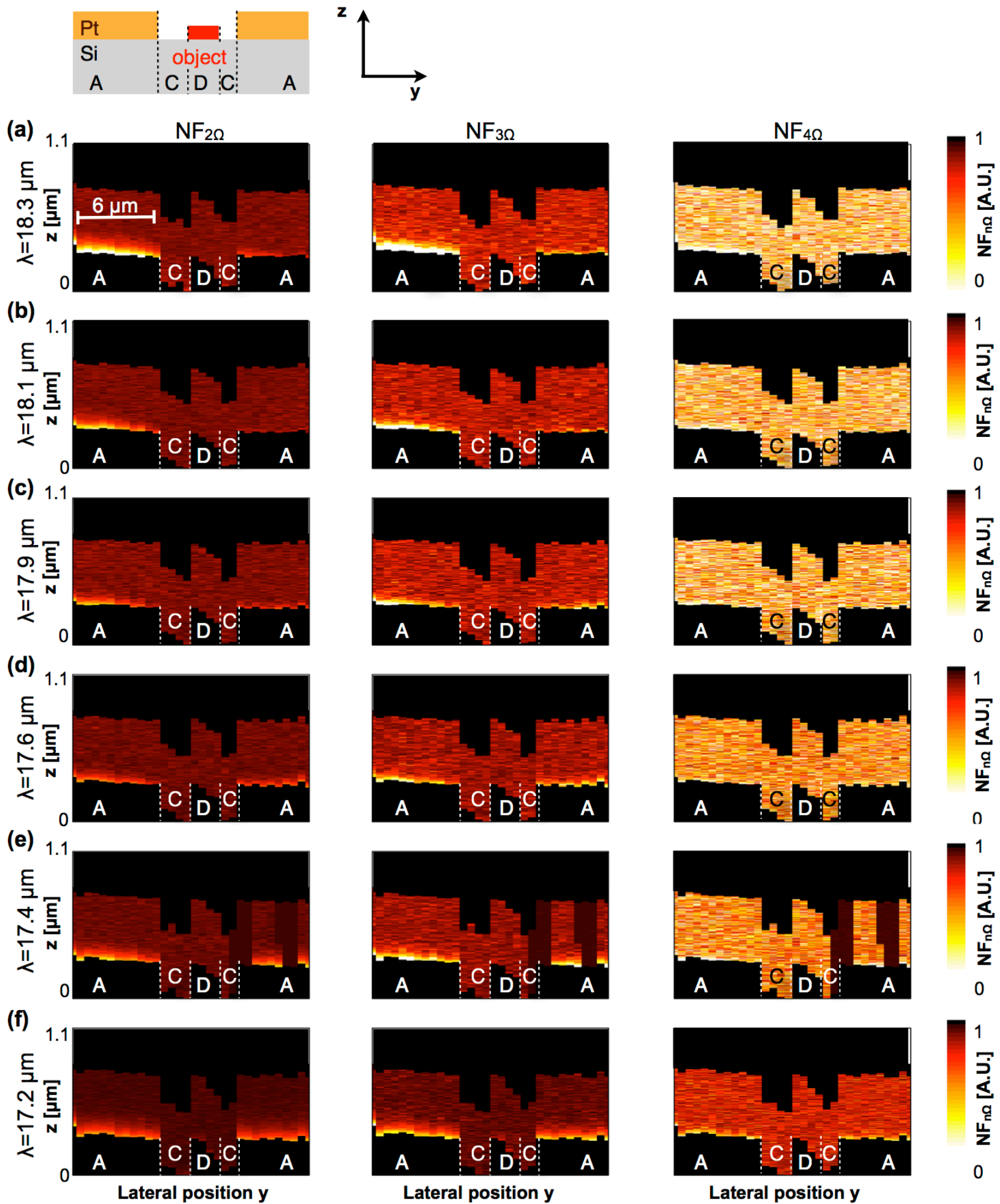

Figure S4 - Part 1 I Near-field cross sections along the reference structure for wavelengths between (a) $18.3 \mu \mathrm{m}$ to (f) $17.2 \mu \mathrm{m}$. Data is displayed for $2^{\text {nd }}, 3^{\text {rd }}$ and $4^{\text {th }}$ harmonic demodulation and is normalized to the incident laser power, and is displayed in the same color scales for same harmonics. The dark vertical lines in (e) correspond to laser failure during recording. For the reference structure the near field is always strongest on the Pt film (A). 

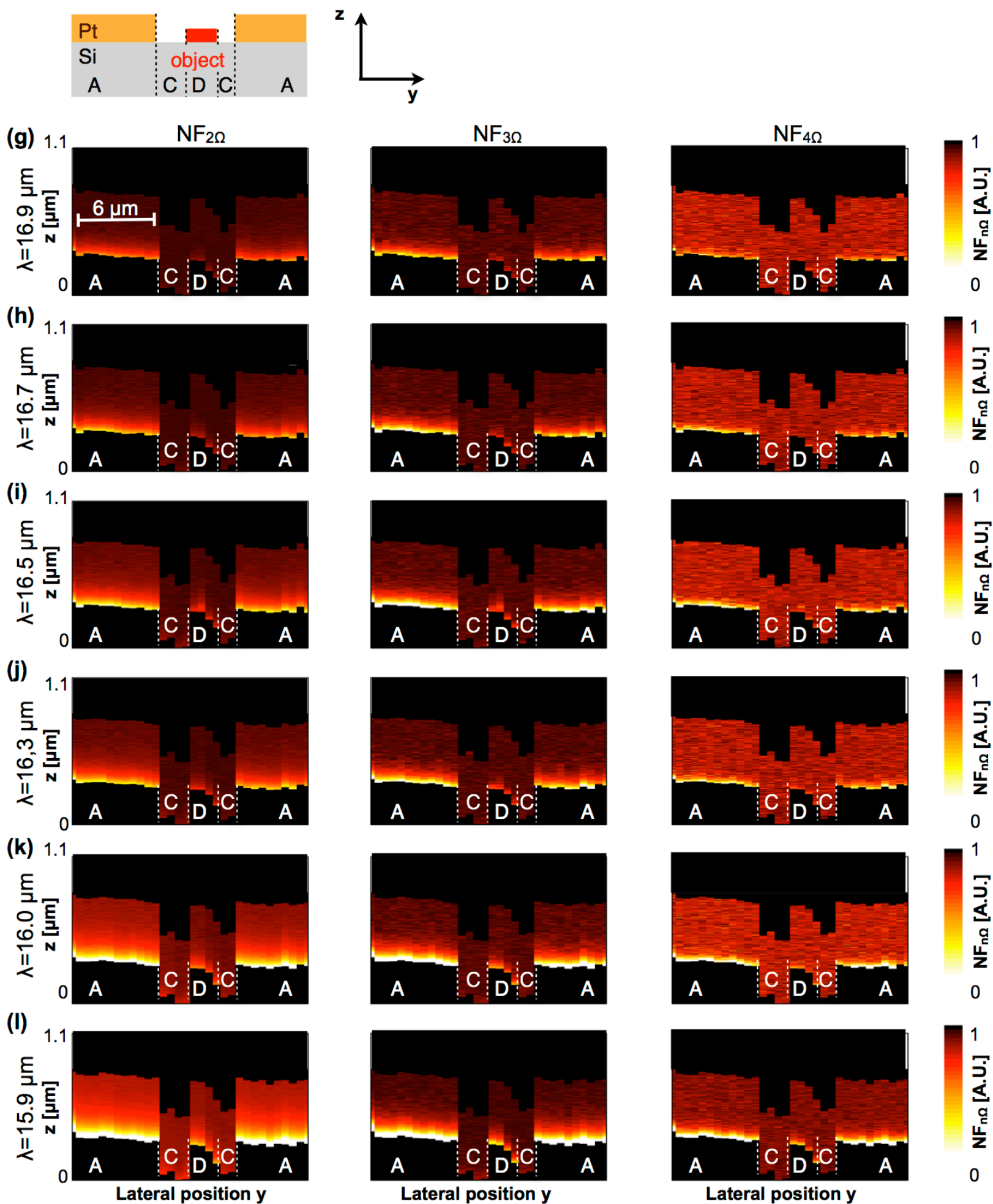

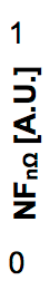

Figure S4 - Part 2 I Near-field cross sections along the reference structure for wavelengths between (a) $16.9 \mu \mathrm{m}$ to (f) $15.9 \mu \mathrm{m}$. Data is displayed for $2^{\text {nd }}, 3^{\text {rd }}$ and $4^{\text {th }}$ harmonic demodulation and is normalized to the incident laser power and is displayed in the same color scales for same harmonics. For the reference structure, the near-field is strongest on the platinum film $(A)$ and somewhat weaker for the structured platinum in the center (D). 